\title{
The rebirth of neuroendoscopic choroid plexus coagulation as treatment of pediatric hydrocephalus
}

\author{
Abbreviations: ETV, endoscopic third ventriculostomy; CPC, \\ choroid plexus coagulation
}

\section{Editorial}

Pediatric hydrocephalus is one of the most frequently diagnosed diseases in pediatric neurosurgical practice. Approximately $60 \%$ of the total cases of hydrocephalus in children are congenital or acquired in childhood. It is a typical surgical disease and if left untreated, most cases are lethal. Traditionally, shunts are the main treatment since 1940s. However, sometimes shunt dependency in children with hydrocephalus is a terrible problem, and is more dangerous in emerging countries than developed countries because of the difficulties that prevent access to prompt neurosurgical intervention, in cases of infection or shunt malfunction. In this scenario, mainly in obstructive hydrocephalus, Neuroendoscopic techniques such as Endoscopic Third Ventriculostomy (ETV) become vitally important in the management of these patients.

ETV is successful in about $80 \%$ of children older than 1year of age, regardless of the cause of obstructive hydrocephalus. For infants younger than 1 year of age, isolated ETV remains controversial, probably because of a deficiency in CSF absorption. Benjamin C. Warf, at CURE Children's Hospital of Uganda, hypothesized that the addition of Neuroendoscopic bilateral Choroid Plexus Coagulation (CPC) at the time of the ETV, to simultaneously reduce the rate of CSF production, might improve the outcome. ${ }^{1}$ This old Neuroendoscopic procedure of the early 20 th century has been reinvented and adapted to current neurosurgery.

Historically, isolated Neuroendoscopic CPC was first described by Putnam in 1934. In 1970, Scarff published the first large series of CPC cases, his own series of 39 children treated during 23-year period, with $67 \%$ of success. After the report of Milhorat, 1974, that CPC in rhesus monkeys reduced CSF production by only $40 \%$, it declined in favor of the shunts. In 1995, Pople and Etles reported the results of CPC in 104 patients, showing that success rate was significantly higher in infants with communicating hydrocephalus. ${ }^{2}$ In fact, these first experiences were quite controversial, perhaps because of technological limitations.

In the early 2000s, Warf's Uganda series for the first time investigated the benefic effect of ETV associated to CPC, again arousing interest in this technique. This study concluded that ETV/CPC procedure is superior to ETV alone in infants younger than 1year of age, particularly among those with nonpost infectious hydrocephalus and myelomeningocele, but longer follow-up with neurocognitive assessment is necessary. ${ }^{1}$ The same series has also defended the use of the ETV/CPC for communicating hydrocephalus. ${ }^{3} \mathrm{ETV} / \mathrm{CPC}$ was significantly more successful than ETV alone in treating congenital idiopathic hydrocephalus of infancy. The primary effect of ETV, as a pulsation absorber, and of $\mathrm{CPC}$, as a pulsation reducer, may be to reduce the net force of intraventricular pulsations that produce ventricular expansion. On the other hand, ETV alone may be less successful for infants because of greater brain compliance. Therefore, ETV/CPC should be considered an effective primary treatment option.

\author{
Volume I Issue 3 - 2014 \\ Roberto Alexandre Dezena \\ Division of Neurosurgery, Federal University of Triangulo \\ Mineiro, Brazil
}

Correspondence: Roberto Alexandre Dezena, Division of Neurosurgery, Clinics Hospital, Federal University of Triangulo Mineiro, Getulio Guarita, I3, Postal Code 3825-44- Uberaba, Minas Gerais, Brazil, Tel +55-343-318-5320,

Email rdezena@yahoo.com.br

Received: June 25, 2014 | Published: July 0I, 2014

Dandy-Walker complex is another condition treatable by ETV/CPC. ${ }^{4}$ Inside also Uganda scenario it was the larger series of this disease treated by Neuroendoscopy and this technique should be strongly considered as the primary management in place of the traditional standard of creating shunt dependence.

Besides ETV/CPC combined technique, nowadays there are new indications for isolated $\mathrm{CPC}$, such as in extreme hydrocephalus and hydranencephaly. ${ }^{2}$ Avoidance of a CSF shunt is desirable in these conditions, since the thinness and fragility of their scalp, besides the common presence of infected scalp ulcers at the parietal bosses. This African study concluded that isolated CPC stabilizes macrocephaly in approximately $40 \%$ of infants and can be considered as an alternative to VP shunt placement.

Unfortunately, it's not known about the long-term effects of coagulating about $80 \%$ of a child's choroid plexus and leaving larger ventricles, associated or not to ETV, specially in terms of neurocognitive aspects. However, it is a promising way to improve outcome of hydrocephalus, reducing shunt dependency, mainly in emerging countries. More studies with larger series are necessary to define the evident benefits of this technique.

\section{Acknowledgments}

None.

\section{Conflicts of interest}

The authors declare there are no conflicts of interest related to the article.

\section{References}

1. Warf BC. Comparison of endoscopic third ventriculostomy alone and combined with choroid plexus cauterization in infants younger than 1 year of age:a prospective study in 550 African children. $J$ Neurosurg. 2005;103(6 Suppl):475-481. 
2. Shitsama S, Wittayanakorn N, Okechi H, et al. Choroid plexus coagulation in infants with extreme hydrocephalus or hydranencephaly. J Neurosurg Pediatr. 2014;14(1):55-57.

3. Warf BC. Congenital idiopathic hydrocephalus of infancy:the results of treatment by endoscopic third ventriculostomy with or without choroid plexus cauterization and suggestions for how it works. Childs Nerv Syst. 2013;29(6):935-940
4. Warf BC, Dewan M, Mugamba J. Management of Dandy-Walker complex-associated infant hydrocephalus by combined endoscopic third ventriculostomy and choroid plexus cauterization. J Neurosurg Pediatr. $2011 ; 8(4): 377-383$. 